\title{
Signaling about norms: Socialization under strategic uncertainty*
}

\author{
Fabrizio Adriani ${ }^{1}$ and Silvia Sonderegger ${ }^{2}$ \\ ${ }^{1}$ University of Leicester, University Road, Leicester, LE17RH, UK fa148@le.ac.uk \\ ${ }^{2}$ University of Nottingham and CeDEx, University Park, Nottingham, NG72QX, UK \\ silvia.sonderegger@nottingham.ac.uk
}

October 17, 2016

\begin{abstract}
We consider a signaling model where adults possess information about the dominant social norm. Children want to conform to whatever norm is dominant but, lacking accurate information, take the observed behavior of their parent as representative. We show that this causes a signaling distortion in adult behavior, even in the absence of conflicts of interest. Parents adopt attitudes that encourage their children to behave in a socially safe way, i.e. the way that would be optimal under maximum uncertainty about the prevailing social norm. We discuss applications to sexual attitudes, collective reputation, and trust.
\end{abstract}

JEL codes: C72, D83, D80, Z13. Keywords: Signaling, Social Norms, Cultural Transmission, Complementarities, Conformity.

\section{Introduction}

Family background affects disparate types of behavior ranging from marriage choices and political attitudes to human capital investment and civic engagement. The role of parental influence in determining child behavior is the object of countless studies across several disciplines. Within economics, a literature started by the seminal paper of Bisin and Verdier (2001) focuses on the intergenerational transmission of culture. ${ }^{1}$ The main innovation of this literature is the

\footnotetext{
${ }^{*}$ We thank the editor, Peter Sørensen, and two anonymous referees for their constructive comments which have helped to improve the paper. We are indebted to Chris Wallace for suggestions given at various stages of the project and to David Myatt for pointing out that a global games approach could be fruitfully applied to our problem.

${ }^{1}$ The cultural evolution literature originally started outside economics, with the of Cavalli-Sforza and Feldman (1981) and Boyd and Richerson (1985). Bisin and Verdier (2010) provide a recent survey.
} 
reckoning that the socialization choices made by parents are shaped by strategic motives. This literature, however, tends to emphasize the long term dynamics of cultural evolution, leaving the mechanism of transmission in the background. In this paper, we retain the idea that parents are strategic but take a different perspective. We focus on a precise mechanism of parent-child socialization, which takes the form of signaling. The underlying idea is simple: parents possess superior information about the surrounding environment. Children thus (consciously or unconsciously, through an "as if" process) shape their beliefs about society on the behavior of their parents.

Recent field and experimental evidence shows that parents distort their behavior when observed by their children. Houser et al. (2016) find that the tendency of parents to cheat in a coin-toss experiment is significantly diminished when their child is in the room. Similarly, Ben-Ner et al. (2015) report that parents increase their contributions in the dictator game when these will be shown to their children. ${ }^{2}$ This "audience effect" suggests that signaling may play an important role in parent-child socialization.

The aim of our work is to understand how parent-child signaling affects social norms. We consider a setting with a continuum of partially informed players (adults) and uninformed players (children). Each adult has altruistic motives toward his own child. Adults face a binary choice between two variants of a cultural trait. After observing their parent's action, each child also faces a binary choice. An obvious example is one where children must also choose which cultural trait to adopt, although as we will see our setup is more general and encompasses other applications.

An essential ingredient of our model is that the child's utility from either choice depends on the dominant social norm. This is for instance the case when there are economic gains from adopting the prevalent cultural variant, or in the presence of a "conformist bias" such as that proposed by Boyd and Richerson's (1985) theory of culture. For example, honest behavior is typically more appealing when a social norm for honesty is prevalent. Children form beliefs about the dominant social norm by observing the behavior of their parents. The signaling game we consider is thus somewhat anomalous, since senders (parents) convey information about aggregate adult behavior in society (i.e., the dominant norm), rather than signaling their own

\footnotetext{
${ }^{2}$ The study also identifies what the authors call an "emulation effect": after observing the choice of their parent (or another adult), children modify their giving in the dictator game, in order to narrow the difference. This suggests that children actively mould their behavior on that of adults around them.
} 
characteristics.

The second crucial ingredient in our story is the presence of strategic uncertainty - i.e., uncertainty concerning the behavior and beliefs (and beliefs about the beliefs) of others. To model strategic uncertainty, we borrow from the Macro-finance literature and adopt a global games approach. ${ }^{3}$ As argued by Morris and Shin (2003), "the apparent indeterminacy of beliefs [that arises in many settings] can be seen as the consequence of two modeling assumptions introduced to simplify the theory. First, the economic fundamentals are assumed to be common knowledge; and second, economic agents are assumed to be certain about each other's behavior in equilibrium." Global games relax these assumptions by modelling information in a more realistic way, thereby escaping the indeterminacy straitjacket.

More concretely, in our setup aggregate behavior is not directly observable. All adults receive private but correlated signals about the underlying environment, and use their private information to predict the information available to others - and thus, their behavior. As in all global games, each adult's information can be very accurate, so long as it is not perfect. As we will see, this "grain of doubt" over the behavior of others allows to pin down precisely which self-fulfilling beliefs will prevail. In this respect, our model thus allows to gain insight into a notoriously elusive issue, namely the nature of the norm that emerges in equilibrium.

We show that the desire to send the "right" signal to their child causes adults to distort their behavior. We call this distortion the norm-signaling bias. Intuitively, if the child knew the norm, then each adult could simply take the action that maximizes his own expected utility. By converse, with signaling, adults have to take into account the fact that their behavior might mislead their child into adopting the "wrong" behavior. Interestingly, we show that this bias arises even when the interests of adults and children are perfectly aligned. ${ }^{4}$

Our setup allows us to characterize the signaling distortion in a precise manner. To fix ideas, suppose that both parent and child must choose between being honest or dishonest. If all parents are honest, a child's best reply is to be honest (e.g., because dishonesty would attract severe sanctions or ostracism), while if all parents are dishonest then dishonesty is optimal for the child too. Consider now a society that is split half/half between honest and dishonest.

\footnotetext{
${ }^{3}$ More precisely, our model draws on the literature on binary action global games (Carlsson and van Damme, 1993, Morris and Shin, 1998).

${ }^{4}$ Our setup thus dispenses with what Bisin and Verdier (2001) call "imperfect empathy", namely the idea that parents may want their children to adopt a certain cultural trait (i.e., their own) even if this is not in the child's interest.
} 
What is the child's optimal behavior in that case? The answer to this question identifies what we call the socially safe choice for the child. This is the action that would be optimal for the child under maximum strategic uncertainty. We show that signaling always induces parents to tilt their behavior in a way that encourages their child to take the socially safe choice. In the simple illustration above, if honesty is socially safe for the child, then the bias will take the form of parents adopting the behavior that encourages their children to behave honestly - in the example, this means being more honest themselves. Furthermore, the parents' propensity to behave honestly will increase as their behavior becomes more visible to their children.

What is the rationale behind the form taken by the signaling bias? The intuition relies on parents using their own private signal about the underlying environment to form expectations about the signals received by others. Each parent (rationally) thinks he is fully representative of the overall population - in fact, he believes his signal to be the median signal. This implies that the adult with the marginal signal (i.e., who is indifferent between the two actions) believes behavior in the population to be split half/half. This is a consequence of the so-called Laplacian Property of global games. As a result of his beliefs, the marginal adult distorts his behavior to encourage his child to choose the action that he (the marginal adult) believes to be optimal, i.e. the socially safe choice. As is usual in economics, what happens at the margin determines overall behavior. Note that, in our model (and, generally, in global games), agents try to predict the information available to others (and thus their beliefs) by taking their own information as representative. While in our case this follows from standard Bayesian updating, the resulting effect mimics well documented psychological tendencies, whereby people tend to overstate the extent to which their attitudes and beliefs are representative of the overall population. A case in point is the concept of false consensus (Ross et al. 1977) and the related notion of self-similarity (Rubinstein and Salant, 2016). ${ }^{5}$

Finally, an equivalent way to identify the socially safe choice in practice is to look at which type of miscoordination would be more costly for the child - what would be worse, to be dishonest when all adults are honest, or to be honest in a dishonest world? The socially safe choice minimizes this possible cost. This implies that social norms will be partly shaped by the adults' desire to shelter their child from the potential costs of miscoordination, and may explain for instance why parents often adopt behavior aimed at promoting a certain degree of "caution" in their children.

\footnotetext{
${ }^{5}$ See Marks and Miller (1987) for a survey of the large psychology literature on the false consensus effect.
} 
The second part of the paper discusses three applications that illustrate the socio-economic implications of the norm-signaling bias. In the first, parents select their attitude on sex issues and children choose whether to engage in pre-marital sex or not. We show that the equilibrium is characterized by hypocrisy. When abstinence is socially safe, instances may arise where all parents have liberal views (and know that all parents have liberal views), but still adopt a conservative attitude. This happens because the need to signal to children that there is a norm against pre-marital sex forces parents to take a conservative attitude, thus helping to preserve the norm against pre-marital sex. We show that hypocrisy is more likely to arise when parents have full control of the information available to their children, as is for instance the case with home schooling.

The second application considers collective reputation problems (Arrow, 1973; Coate and Loury, 1993; Saez-Martí and Zenou, 2012). In a simplified version of Coate and Loury's (1993) model, we investigate under what conditions it is optimal for members of a given social group to socialize their children to work hard. In equilibrium, children with strong work norms tend to invest more in skill acquisition. In environments conducive to low social mobility (e.g. where access to education is expensive for group members), not investing is socially safe. Accordingly, parents choose to instil a weak work norm in order to signal to the child that he should not waste resources in trying to climb the social ladder, thus helping to preserve the lack of social mobility. By contrast, in environments where education is easily accessible to all, investing is socially safe and strong work norms emerge in equilibrium. Our model also suggests that social mobility is a substitute for monetary incentives in shaping work norms. In societies with high mobility, strong work norms emerge even in the absence of high powered incentives.

Following Adriani and Sonderegger (2009), the third application looks at under what conditions parents choose to instil pro-social values into their children. We show that this happens when the returns from economic exchange are high. By raising their children as pro-social, parents signal that they are optimistic about the prevalence of trustworthiness in society, and thus encourage their children to exhibit trust in economic exchanges. Since other parents do the same, this creates a virtuous cycle. The final outcome is that the actual rewards from trusting others outweigh the forgone rents from dishonesty.

Related literature As already mentioned, the literature on cultural transmission is primarily concerned with the dynamics of cultural evolution. Prominent examples include Hauk and SaezMartí (2002), Kuran and Sandholm (2008), and Tabellini (2008). These papers study how the 
distribution of given cultural traits in the population (e.g. corruption, cooperation) evolves over time. The cultural transmission mechanism is typically modelled with a reduced form approach following Bisin and Verdier (2001). Our focus is different: we analyze an explicit signaling mechanism for parent-child socialization. Signaling has a long tradition in economics, and a number of works have explored culture and social norms through the lens of information transmission although not parent-child signaling. Examples in this direction include Bernheim's (1994) seminal work on conformity, Bénabou and Tirole's (2006) and Ellingsen and Johannesson's (2008) analysis of prosociality, Levy and Razin's $(2012,2014)$ study of religious behavior and Fang's (2001) model of élite cultural activities. In all these works, agents are heterogeneous in some unobservable attribute and are concerned about society's perception of their type. They thus adopt a certain cultural attitude to signal to others that they possess desirable personal characteristics. In our setup, by contrast, the signal conveys information about the dominant norm. A few papers in the literature have explicitly studied norm-signaling, although within setups that differ fundamentally from ours. In Sliwka (2006), an employer's contract signals to potential employees the nature of the dominant norm in the workplace. In Acemoglu and Jackson (2015), agents gain information about norms by looking at the recent history of play. In Bénabou and Tirole (2011), laws are informative of the prevailing behavior in society. Our setup is also related to the leading-by-example model by Hermalin (1998), where the leader's choice of effort is used by followers to make inferences about the returns from a given activity. Among other differences, all these works lack an essential ingredient of our setup, namely altruistic motives by the sender toward the receiver.

The paper is organized as follows. Section 2 presents and analyzes a simplified two-generation version of the model. Section 3 considers a more general OLG structure that incorporates a number of natural extensions. The literature on cultural transmission (such as Bisin and Verdier, 2001, Saez-Martí and Sjögren, 2008) identifies multiple channels of cultural transmission, such as socialization in the family ("direct vertical socialization") and other socialization processes like social imitation and learning from peers ("oblique/horizontal socialization"). ${ }^{6}$ Accordingly, the general model includes the possibility that children may extract information from non-parental sources, and allows for peer effects both among parents and among children. Section 4 illustrates the effects of the signaling bias in applications. Section 5 addresses further robustness issues and explores additional extensions. In particular, we argue that the framework is flexible enough

\footnotetext{
${ }^{6}$ The vertical/horizontal terminology was first introduced by Cavalli-Sforza and Feldman (1981).
} 
to accommodate other aspects of cultural transmission, such as cultural persistence and path dependence. We also discuss the robustness of the signaling equilibrium to cheap talk. Section 6 concludes. All proofs not included in the text can be found in the supplementary material.

\section{Baseline Model}

Most of our results are driven by a single effect. For pedagogical purposes, we start off with an extremely stylized setup that allows us to isolate the main force at work from confounding effects. A richer setting is analyzed in Section 3.

Consider an economy populated by a continuum of identical child-adult pairs, indexed by $i \in[0,1]$, where each adult has exactly one child. Adults move first, simultaneously choosing between two variants of a cultural trait, the high action $(a=1)$ and the low action $(a=0)$. The direct economic payoff to an adult from the low action is normalized to zero. The direct payoff from the high action is given by the random variable $\theta$ (the state of nature). We assume that the state of nature is uniformly drawn in the interval $[-D, D]$. Parents do not perfectly observe $\theta$. Each parent $i$ only observes a private noisy signal $\theta_{i}$ uniformly drawn in the interval $[\theta-\epsilon, \theta+\epsilon], \epsilon>0$.

Children simultaneously move after observing the action chosen by their parent. They cannot observe the state of nature, their parent's signal, or the share of adults choosing each action. Each child can choose between a top action $(\alpha=1)$ and a bottom action $(\alpha=0)$. The payoff from the bottom action is zero. The payoff from the top action depends on how the share of adults choosing high, which we denote as $x \in[0,1]$, compares to a "critical share" $r \in[0,1]$. $^{7}$ More precisely, it is given by,

$$
\tilde{v}(x)=\omega(x-r),
$$

where $\omega>0$ is a measure of the strength of oblique complementarities, reflecting the child's concern for selecting the right action given the dominant norm. ${ }^{8}$ Intuitively, (1) captures the idea that selecting top is worthwhile for the child only if there are enough adults selecting high

\footnotetext{
${ }^{7}$ The case where the child's payoff also depends on $\theta$ is addressed below, in Section 4 .

${ }^{8}$ This may arise from concrete economic gains or from conformist tendencies, as in Boyd and Richerson (1985). Note that, although in our theory all children have the same $\omega$, actual societies may display some degree of heterogeneity in how much individuals care about conforming to others. The presence of a minority of children not concerned about conformity (or even with anti-conformist tendencies) would complicate the model without changing the substance of the results.
} 
(i.e. only if their share is at least equal to $r$ ). The value taken by $r$ will depend on the underlying economic environment and is thus application-specific (Section 4 provides some examples). It is important to note that the parameter $r$ is related to the concept of risk dominance developed for $2 \times 2$ games. ${ }^{9}$ This is emphasized by the following definition,

Definition 1. Action $\alpha \in\{0,1\}$ is the socially safe choice if it is optimal for the child under maximum uncertainty over adults' behavior (i.e. if it is optimal when $x$ is uniform in $[0,1]$ ).

Under maximum uncertainty, the child's expected payoff from the top action is $\omega(1 / 2-r)$. The optimal action for the child thus depends on whether $r$ is above or below $1 / 2$. In the former case, the bottom action is optimal (socially safe), while in the latter case the top action is socially safe. For $r=1 / 2$, the two actions have the same safety level.

Intuitively, consider a society where adults are equally split between the two actions. What is optimal for a child in this case? The answer to this question identifies the socially safe action. An equivalent approach is to consider whether it would be worse for a child to be miscoordinated when all adults select low or when all adults select high. The socially safe action minimizes this hypothetical miscoordination cost. ${ }^{10}$

We assume that adults are altruistic toward their children. However, to better compare our setting with a standard signaling game, we allow for the possibility of a conflict of interests between an adult and his child. We model this by assuming that the adult's intrinsic preference over the child's action is given by $\tilde{v}(x)+\beta$, where $\beta \in \mathbb{R}$ measures the conflict of interests. A value of $\beta$ equal to zero means that incentives are perfectly aligned. A positive $\beta$ implies that the parent wants his child to choose the top action in situations where the child would prefer the bottom action (e.g. he does not fully internalize the costs of the top action), while a negative $\beta$ means that the parent is biased against the top action. The role of $\beta$ will be particularly relevant in section 5.2 where we discuss the possibility of parent-child communication via cheap-talk.

Table 1 shows an adult's total utility. More compactly, this can be expressed as

$$
u(x, a, \alpha ; \theta) \equiv a \theta+\alpha[\tilde{v}(x)+\beta]
$$

Discussion Our setup is consistent with a number of possible applications, some of which are discussed in detail in Section 4. Here, we briefly sketch a few additional examples. Tabellini

\footnotetext{
${ }^{9}$ See e.g. Carlsson and van Damme, (1993), and Kandori, Mailath and Rob (1993).

${ }^{10}$ This is because the cost incurred by a child who selects top when $x=0$ is $\omega r$, while the (opportunity) cost incurred by a child who selects bottom when $x=1$ is $\omega(1-r)$.
} 


\begin{tabular}{cccc}
\hline & & \multicolumn{2}{c}{ Child's action } \\
& & Bottom & Top \\
\hline \multirow{2}{*}{ Adult's action } & Low & 0 & {$[\tilde{v}(x)+\beta]$} \\
& High & $\theta$ & $\theta+[\tilde{v}(x)+\beta]$ \\
\hline
\end{tabular}

Table 1: Total parental utility (terms in brackets represent parent's indirect utility from child's choice).

(2008) studies the intergenerational transmission of honesty/dishonesty. In his model, the reward from adopting honesty as a cultural trait essentially depends on two variables: the quality of institutions and how widespread honesty is in society. In the baseline model presented above, the first variable is captured by $\theta$, while the second is given by $x \cdot{ }^{11}$ Different from standard theory, however, the quality of institutions $(\theta)$ is not common knowledge in our setting. This in turn generates uncertainty about the prevalence of honesty $(x)$. Note also that, for the sake of simplicity, we assume that the parents' direct payoff from honesty only depends on $\theta$, while the children's payoff only depends on $x$. The more general model of Section 3 allows both payoffs to depend on both variables. The insights generated by our theory can also be used to study aspects of social behavior like religiousness, dress codes and eating, drinking or sexual practices. The setup may also apply to environments where the terminology "parents" and "children" applies metaphorically. A case in point is that of an academic advisor and a $\mathrm{PhD}$ student.

Finally, it is worth noting that our model can be extended to the case where children do not care about the fraction of adults who adopt a given behavior but care instead about the fraction of adults who hold particular beliefs about $\theta$. For instance, children may worry about the fraction of adults who believe that social drinking is fundamentally wrong independently of the extent to which adults engage in social drinking. This setting would yield results that are quantitatively smaller but qualitatively similar to ours.

\subsection{Analysis}

Consider first the benchmark case where children are able to perfectly observe $x$. In that case, the child's equilibrium strategy consists in choosing the top action whenever $x \geq r$ and, crucially, is independent of parental behavior. It is then clear that, with no signaling concerns, each parent

\footnotetext{
${ }^{11}$ Other features of Tabellini (2008) find parallels in our setting. For instance, the fact that parents may want their children to adopt traits that do not necessarily maximize their material welfare (imperfect empathy) is loosely captured in our model by the "conflict of interests" parameter $\beta$.
} 
will choose the high action when $\theta_{i} \geq 0$ and the low action when $\theta_{i}<0 .{ }^{12}$

Consider now the more interesting case where children do not observe $x$, but only the action chosen by their own parent. This opens the door to the possibility of parent-child signaling. Notice that parents do not directly observe the behavior of other adults. However, a parent's private signal $\theta_{i}$ is informative of the distribution of beliefs in the adult population. This gives the parent an informational advantage over the child in predicting aggregate adult behavior $(x)$.

Parent-child signaling affects the nature of strategic interactions among parents in a fundamental way. To see how this happens, let $\alpha(a), \alpha:\{0,1\} \rightarrow[0,1]$ denote the probability that a child chooses the top action upon observing his parent choosing action $a \in\{0,1\}$. The values of $\alpha(1)$ and $\alpha(0)$ are determined in equilibrium and reflect the extent to which a parent's action affects his child's choice. The parent's payoff from the high action is then $\theta+[\tilde{v}(x)+\beta] \alpha(1)$, while that from the low action is $[\tilde{v}(x)+\beta] \alpha(0)$. Letting $\Delta \alpha \equiv \alpha(1)-\alpha(0)$, the net payoff from the high action can be expressed as

$$
\theta+[\tilde{v}(x)+\beta] \Delta \alpha
$$

We refer to (3) as the parent's inclusive payoff. Differentiating the term in brackets with respect to $x$ yields $\omega \Delta \alpha$. Whenever $\Delta \alpha>0$ a parent's incentive to choose the high action is (weakly) increasing in the share of parents who choose the same action. Intuitively, the higher parent $i$ 's propensity to choose the high action, the higher the incentive for parent $j$ to do the same to signal to his child that the high action is prevalent in society. This stands in contrast with the case of informed children, where, by construction, there is no complementarity in adult behavior. The difference between the two cases emerges even though payoffs are exactly the same - the only difference is in the information structure. This suggests that norm signaling affects the nature of the equilibrium by inducing a "spurious" complementarity in adults' behavior.

If the state $\theta$ were publicly observable, then the presence of complementarity would mean that, for values of $\theta$, adults' behavior would not uniquely determined - that is, even keeping children's strategies fixed. However, since information on $\theta$ is private and noisy, adults lack common knowledge of the game being played: only the fact that $\theta \in[-D, D]$ is common knowledge. As in all global games, if the support of $\theta$ is wide enough, then an unravelling process comes into effect. In particular, an adult observing a signal sufficiently close to the

\footnotetext{
${ }^{12}$ Throughout the paper, we use the convention that a parent who is indifferent chooses the high action.
} 
upper (lower) bound of the support will find it optimal to play $a=1(a=0)$ independently of what other adults do. Acknowledging this, someone with a slightly less extreme signal will adopt the same behavior. This in turn induces someone with an even less extreme signal to conform and so on. Applying this argument iteratively yields a unique threshold value of the signal, $k$, such that adults choose $a=1$ if $\theta_{i} \geq k$ and $a=0$ otherwise. The distinct feature of our setting is that all this is driven by signaling. Adults adopt a given behavior to signal to their children that that behavior is likely to be prevalent. By doing this, they make that behavior more widespread, and thus signaling more compelling.

For the purposes of this simple illustration, we restrict attention to Perfect Bayesian Equilibria $(\mathrm{PBE})$ where all adults follow a threshold strategy of the type described above. For technical reasons, we will only consider parameter configurations yielding an equilibrium cutoff that is more than $3 \epsilon$ away from the bounds of the support of $\theta$, i.e. $k \in[-D+3 \epsilon, D-3 \epsilon] .{ }^{13}$ We will refer to a cutoff satisfying this condition as sufficiently interior. Denote with $x(k)$ the share of adults choosing the high action. The equilibrium characterization proceeds through the following four steps:

1. Given any $\epsilon>0$, there exists $D$ sufficiently large such that $\Delta \alpha=1$, i.e. it is optimal for children to choose the top action if and only if they observe their parent choosing the high action. This result is established more generally in the next section. Intuitively, the larger is $D$ relative to $\epsilon$, the more parental behavior is informative about the behavior of other adults and thus the stronger the child's incentive to follow it.

2. Given $\Delta \alpha=1$ and taking expectations in (3), a parent observing signal $\theta_{i}$ will choose the high action whenever

$$
E\left[\theta \mid \theta_{i}\right]+\omega\left(E\left[x(k) \mid \theta_{i}\right]-r\right)+\beta \geq 0 .
$$

When $k$ is sufficiently interior, $E\left[x(k) \mid \theta_{i}\right]$ is a non-decreasing function of $\theta_{i}{ }^{14}$ Since $E\left[\theta \mid \theta_{i}\right]$ is increasing, this ensures that a threshold strategy is optimal for each individual adult.

3. Any adult with $\theta_{i} \in[-D+\epsilon, D-\epsilon]$ believes that the distribution of signals of other adults is centred around his own realization. This implies that the marginal parent (with

\footnotetext{
${ }^{13}$ Generally speaking, for the Laplacian property to apply, the cutoff must be at least $\epsilon$ away from the bounds of the support. The stricter requirement that $k$ be at least $3 \epsilon$ away from the bounds makes it easier to establish monotonicity of the adults' expected payoff.

${ }^{14}$ This is formally established in the supplementary material, Section A.1.
} 
$\left.\theta_{i}=k\right)$ has Laplacian posterior beliefs over $x(k)$. To show this, note that, given any $\theta \in[k-\epsilon, k+\epsilon]$,

$$
x(k)=\int_{k}^{\theta+\epsilon} \frac{1}{2 \epsilon} d \theta_{i}=\frac{1}{2}+\frac{\theta-k}{2 \epsilon} .
$$

Since $\theta$ is, conditional on $\theta_{i}=k$, uniformly distributed in $[k-\epsilon, k+\epsilon]$, the marginal parent believes that $x(k)$ is uniform in $[0,1] .{ }^{15}$

4. Given his beliefs about $x$, the marginal parent must be indifferent between the two actions. This implies that, evaluated at $\theta_{i}=k,(4)$ must be zero. Imposing $E[x(k) \mid k]=1 / 2$, $E[\theta \mid k]=k$, and solving for $k$ yields $k=\theta^{*}$, where

$$
\theta^{*}=\underbrace{-\beta}_{\text {Conflict of interest }}+\underbrace{\omega\left(r-\frac{1}{2}\right)}_{\text {Norm-signaling bias }} .
$$

The argument is then concluded by noting that $\theta^{*}$ does not depend on $D$ and the requirement of a sufficiently interior cutoff reduces to $D \geq\left|\theta^{*}\right|+3 \epsilon$.

Proposition 1. For D sufficiently large, the unique threshold PBE is such that each parent $i$ chooses $a=1$ if $\theta_{i}$ exceeds a cutoff $\theta^{*}$ given by (6) and chooses $a=0$ otherwise. Children conform to observed adult behavior by choosing $\Delta \alpha=1$.

A number of remarks are in order. As already noted, if children perfectly knew $x$, adults would have a cutoff equal to zero. Expression (6) thus measures the distortion due to signaling. This has two components. The first, captured by the term $\beta$, reflects the conflict of interests between parents and children. This distortion arises because there are situations where the parent would like the child to choose one action, but the child would prefer the other. The second component, the term $\omega(r-1 / 2)$, is what we call the norm-signaling bias. This bias emerges also when the interests of parents and children are perfectly aligned $(\beta=0)$. The distortion arises because parents anticipate that their children will infer the dominant norm from their behavior. Since parents are altruistic, they internalize the information externality that they impose on their children. The expression $\omega(r-1 / 2)$ makes it transparent that the norm-signaling bias goes in the direction of encouraging the child to select his socially safe

\footnotetext{
${ }^{15}$ It is worth stressing that the assumption of uniform distributions is not critical for this step. Indeed, a Laplacian posterior would also emerge in a model with a normal signal and an uninformative prior and can be seen as an approximation of any model where the adults' signal is very precise vis-á-vis the prior (see Morris and Shin, 2003).
} 
choice. When the top action is socially safe $(r<1 / 2)$, the bias is negative, since parents are more inclined to select the high action (to induce their children to choose the top action). The reverse occurs when the socially safe choice is the bottom action. Note that, although the socially safe choice determines the direction of the norm signaling bias, its magnitude depends on the strength of oblique complementarities $(\omega)$. Intuitively, the larger the sensitivity of the child's payoff to the dominant social norm, the higher the propensity of the parent to distort his behavior to shelter his child from costly mistakes.

Another worthwhile observation is that step 3 of the proof relies on parents using their own signal to form expectations about the signals received by others. In fact, each parent believes his own signal to be the median signal in the population. As explained in step 4, this feature is instrumental in pinning down the unique equilibrium cutoff. In our setup (and, generally, in global games), this is fully consistent with rational Bayesian updating. However, there are clear parallels with so-called behavioral biases, notably the false-consensus effect, widely acknowledged in psychology. This is an egocentric bias that captures the tendency of people to believe their opinions, beliefs, preferences etc. are "normal" and fairly typical of those of others. ${ }^{16}$

\section{$3 \quad$ Full model}

We now extend the results of Proposition 1 in several directions. First, we allow the child's payoff to also depend on the state of nature. This implies that the parent's motives for signaling are now mixed: Parental behavior conveys information both about the social norm and about the state of nature. Second, we consider the case where children may obtain information from nonparental sources. Third, we allow for strategic interactions both with peers and with members of the other generation. Fourth, we include pure externalities in order to highlight some welfare implications of norm signaling.

We start off by embedding the simple model seen in the previous section in an overlapping generation framework. More precisely, in every period $t$,

1. Every $t-1$ child becomes adult and gives birth to one child.

2. Nature draws an iid state $\theta_{t} \sim[-D, D]$.

\footnotetext{
${ }^{16}$ The consensus effect has long been recognized by psychologists, at least since the seminal paper by Ross et al. (1977). Economists have also increasingly started to document and to pay attention to this phenomenon. See, e.g., Adriani and Sonderegger (2015) for a theoretical analysis.
} 
3. Each adult observes a private noisy signal $\theta_{i t} \sim U\left[\theta_{t}-\epsilon, \theta_{t}+\epsilon\right]$ and selects his action $a$.

4. Children observe the action of an adult, either their parent or someone else (more below), and select their own action $\alpha$.

In period $t+1$, each time $t$ child becomes adult and gives birth to one child, a new state of nature $\theta_{t+1} \sim U[-D, D]$ is drawn and so on. While the setting discussed in this section is essentially a repeated/stationary version of the two stage example seen above, we discuss in Section 5 how to incorporate sources of persistence and path dependence. Let

$$
\mathbf{x}_{t} \equiv\left[\begin{array}{c}
x_{t} \\
\tilde{x}_{t}
\end{array}\right], \boldsymbol{\omega}_{p} \equiv\left[\begin{array}{l}
\omega_{p p} \\
\omega_{p c}
\end{array}\right], \boldsymbol{\omega}_{c} \equiv\left[\begin{array}{l}
\omega_{c p} \\
\omega_{c c}
\end{array}\right], \mathbf{r}_{p} \equiv\left[\begin{array}{c}
r_{p p} \\
r_{p c}
\end{array}\right], \mathbf{r}_{c} \equiv\left[\begin{array}{l}
r_{c p} \\
r_{c c}
\end{array}\right]
$$

where $x_{t}$ and $\tilde{x}_{t}$ (both in $[0,1]$ ) denote the fractions of parents choosing the high action and children choosing the top action, respectively. All other symbols denote constant parameters (details below). A parent's direct payoff is now,

$$
v\left(\mathbf{x}_{t} ; \theta_{t}\right)=\boldsymbol{\omega}_{p}^{\prime}\left(\mathbf{x}_{t}-\mathbf{r}_{p}\right)+\theta_{t},
$$

when choosing $a=1$ and zero when choosing $a=0$. A child's payoff is

$$
\tilde{v}\left(\mathbf{x}_{t} ; \theta_{t}\right)=\omega_{c}^{\prime}\left(\mathbf{x}_{t}-\mathbf{r}_{c}\right)+l_{\theta} \theta_{t}
$$

when choosing $\alpha=1$ and zero otherwise. An adult's total utility is now given by

$$
u\left(a, \alpha, \mathbf{x}_{t} ; \theta_{t}\right)=a v\left(\mathbf{x}_{t} ; \theta_{t}\right)+\alpha\left[\tilde{v}\left(\mathbf{x}_{t} ; \theta_{t}\right)+\beta\right]+e \mathbf{x}_{t}^{\prime} \mathbf{i},
$$

where $e \in \mathbb{R}$ and $\mathbf{i}=\left(\begin{array}{ll}1 & 1\end{array}\right)^{\prime}$. We now discuss in detail each feature of the extended model.

Mixed motives signaling In some cases, the child's net payoff may also directly depend on the state of nature. To allow for this possibility, we add the term $l_{\theta} \theta_{t}$ in (9), where $l_{\theta} \geq 0$ measures the child's payoff sensitivity to the state of nature. ${ }^{17}$

Non-parental information sources It is natural to assume that children may have direct access to information sources beyond parental control. In order to retain tractability, we model this in a very simple way. We assume that, with probability $\delta \in[0,1]$, the child observes parental

\footnotetext{
${ }^{17}$ The coefficient on $\theta_{t}$ in the parent's payoff is set equal to one. This is an innocuous normalization.
} 
behavior. With the complementary probability, $1-\delta$, the child observes the behavior of another random adult. Adults do not know in advance what their child will observe, but are aware that their actions will provide relevant information to their child with probability $\delta$. Since adults only care about their own child, the benchmark case of no signaling concerns is obtained by setting $\delta=0 .{ }^{18}$ Our qualitative results extend to more complex information structures, where children may be able to observe a number of adults and/or some of their peers, so long as the probability that the parent's behavior is pivotal for his child is strictly positive and sufficiently flat in $\theta_{i}$ for values of $\theta_{i}$ around the cutoff.

Social pressure The payoff specifications (8) and (9) allow for generic interactions. Members of each generation are concerned both about coordinating with their peers and with members of the other generation. If $\omega_{c c}>\omega_{c p}$ and $\omega_{p p}>\omega_{p c}$, then members of each generation are mostly concerned about coordinating with their peers. If $\omega_{c p}>\omega_{c c}$ and $\omega_{p p}>\omega_{p c}$, on the other hand, then all agents are chiefly concerned about coordinating with adults. Child-adult oblique complementarities are captured by the parameter $\omega_{c p}$ in (9). The parameters $r_{c p}, r_{c c}, r_{p p}, r_{p c}$ (all in $[0,1])$ determine which action is socially safe in strategic interactions vis-á-vis each generation. It is also convenient to define four additional parameters: $\bar{r}_{c} \equiv \boldsymbol{\omega}_{c}^{\prime} \mathbf{r}_{c} / \boldsymbol{\omega}_{c}^{\prime} \mathbf{i}, \bar{r}_{p} \equiv \boldsymbol{\omega}_{p}^{\prime} \mathbf{r}_{p} / \boldsymbol{\omega}_{p}^{\prime} \mathbf{i}$, $\bar{\omega}_{c} \equiv \boldsymbol{\omega}_{c}^{\prime} \mathbf{i}$, and $\bar{\omega}_{p} \equiv \boldsymbol{\omega}_{p}^{\prime} \mathbf{i}$. When $\bar{r}_{c}<1 / 2$, the top action is the overall - i.e. taking into account social pressure from both adults and other children - socially safe action for a child. The opposite occurs when $\bar{r}_{c}>1 / 2$. The parameter $\bar{r}_{p}$ plays the same role for parents. The parameters $\bar{\omega}_{c}$ and $\bar{\omega}_{p}$ represent the overall social pressure (from both adults and children) faced by children and adults, respectively.

Consistent with most of the literature on global games, we will predominantly concentrate on the case where $\boldsymbol{\omega}_{c} \geq 0$ and $\boldsymbol{\omega}_{p} \geq 0$ (strategic complementarity). More precisely, the following two assumptions are needed for our results

A1 $\bar{\omega}_{c} \geq 0$ (with strict inequality when $l_{\theta}=0$ ).

$\mathbf{A 2} \bar{\omega}_{p}+\delta \bar{\omega}_{c} \geq 0$.

Assumption A1 ensures that, taking into account interactions with both generations, the child's payoff is on the whole supermodular. The strict inequality when $l_{\theta}=0$ ensures that the

\footnotetext{
${ }^{18}$ In the more plausible scenario where adults also partially care about setting the right example for other adults' children, the signaling distortion would be larger.
} 
child's payoff is non-degenerate. Assumption A2 implies that, once the child's reply is taken into account, the inclusive payoff of each parent is also supermodular. ${ }^{19}$

Pure externalities In some cases, behavior may generate pure externalities, i.e. externalities with no direct consequence for strategic behavior, but with consequences on aggregate welfare. This is captured by the parameter $e \in \mathbb{R}$ in (10). A positive (negative) value of $e$ means that the high action generates a positive (negative) externality. For instance, when $e>0$, the high action can be interpreted as taking an environmentally friendly or a socially engaged attitude/lifestyle.

Relative to the low action, the adult's inclusive net payoff from the high action is now

$$
v\left(\mathbf{x}_{t} ; \theta_{t}\right)+\delta \Delta \alpha\left[\tilde{v}\left(\mathbf{x}_{t} ; \theta_{t}\right)+\beta\right]
$$

where the presence of $\delta$ in (11) reflects the fact that an adult's behavior is now relevant for his child's choice only with probability $\delta$.

\subsection{Full model analysis}

We first characterize the unique Perfect Bayesian Equilibrium within the intuitive class of threshold equilibria where, in each period, adults choose the high action whenever their signal exceeds a cutoff $k$ and children conform to observed adult behavior by choosing $\Delta \alpha=1 .^{20}$ We then provide sufficient conditions for this equilibrium to be the unique of the game.

Consider then a candidate equilibrium with cutoff $k$ and take children first. As noted above, children choose $\Delta \alpha=1$ when the behavior of each adult conveys sufficiently accurate information about the behavior of others and/or of the state of nature. This in turn implies that $\epsilon$ cannot be too large relative to $D$. The next Lemma makes this insight more precise,

Lemma 1. Suppose that parents use an threshold strategy with sufficiently interior cutoff $k$. Then, for any $\epsilon>0$ there exists a $\mathcal{D}(\epsilon)$ such that, if $D>\mathcal{D}(\epsilon), \Delta \alpha=1$. Moreover, $\mathcal{D}: \mathbb{R}^{+} \rightarrow \mathbb{R}^{+}$ is strictly increasing in $\epsilon$ and such that $\lim _{\epsilon \rightarrow 0} \mathcal{D}(\epsilon)=|k|$.

\footnotetext{
${ }^{19}$ Note that supermodularity of the adults' direct payoff (i.e. $\bar{\omega}_{p} \geq 0$ ) is not required. In Section 4.3 we discuss an application where, abstracting from the child's response, parental actions would be strategic substitutes.

${ }^{20}$ Our definition of threshold equilibria involves a slight abuse of terminology. To be precise, we are restricting attention to equilibria where, in all periods, each adult's strategy $a:[-D-\epsilon, D+\epsilon] \rightarrow\{0,1\}$ is a non-decreasing step function of $\theta_{i t}$. We are thus ignoring counterintuitive threshold equilibria with "downward" step functions, where adults switch to the low action as $\theta_{i t}$ increases. As shown below, these can be ruled out under some natural parametric restrictions.
} 
Proof. See supplementary material.

Hence, the larger the heterogeneity in adults' beliefs, $\epsilon$, the wider the support of $\theta_{t}$ must become to ensure that adults' actions are sufficiently informative to sway children's behavior. The expression for $\mathcal{D}$ is not particularly complicated but its properties are more easily derived implicitly. In particular, when $\epsilon$ is vanishingly small, an interior cutoff (i.e. $|k|<D$ ) is alone sufficient to ensure that children conform to observed adults' behavior.

Clearly enough $\Delta \alpha=1$ implies $\tilde{x}_{t}=x_{t}$. Invoking the Laplacian property, one obtains $E\left(\tilde{x}_{t} \mid k\right)=E\left(x_{t} \mid k\right)=1 / 2$. As shown in the Appendix, the conditional expectation of (11) is increasing in $\theta_{i t}$, so that $k$ must satisfy

$$
v\left(\frac{1}{2} \mathbf{i}, k\right)+\delta\left[\tilde{v}\left(\frac{1}{2} \mathbf{i} ; k\right)+\beta\right]=0
$$

Denoting with $\theta^{*}$ the value of $k$ solving (12), one obtains

$$
\theta^{*}=\frac{\bar{\omega}_{p}\left(\bar{r}_{p}-\frac{1}{2}\right)+\delta \bar{\omega}_{c}\left(\bar{r}_{c}-\frac{1}{2}\right)-\delta \beta}{1+l_{\theta} \delta}
$$

Proposition 2. Assume that $\theta^{*}$, as given by (13), is sufficiently interior. If $D>\mathcal{D}(\epsilon)$, then there exists a unique threshold PBE where children conform to observed adult behavior (i.e. choose $\Delta \alpha=1)$. The equilibrium is such that, in every period, adults choose the high action whenever their signal $\theta_{i t}$ is above $\theta^{*}$ and choose the low action otherwise.

Proof. See supplementary material.

We can obtain the cutoff in the absence of signaling concerns, $\theta^{n s}$, by setting $\delta=0$ in (13),

$$
\theta^{n s}=\bar{\omega}_{p}\left(\bar{r}_{p}-\frac{1}{2}\right)
$$

The total distortion due to signaling is thus

$$
\theta^{*}-\theta^{n s}=\frac{\delta}{1+l_{\theta} \delta}\left[-\beta+\bar{\omega}_{c}\left(\bar{r}_{c}-\frac{1}{2}\right)-l_{\theta} \bar{\omega}_{p}\left(\bar{r}_{p}-\frac{1}{2}\right)\right]
$$

To gather intuition, assume first that $l_{\theta}=0$, so that the child's net payoff is not directly affected by the state of nature. Then, the expression for the norm signaling term $\left(\delta \bar{\omega}_{c}\left(\bar{r}_{c}-1 / 2\right)\right)$ becomes very similar to the one obtained in the previous section. The main difference is that the parent now also takes into account his child's desire to coordinate with his peers (since the 
peer pressure term $\omega_{c c}$ enters both $\bar{\omega}_{c}$ and $\bar{r}_{c}$ ). If the socially safe action for children is the same vis-à-vis both adults and other children (e.g. $r_{c c}<1 / 2$ and $r_{c p}<1 / 2$ ), children's peer pressure magnifies the distortion. However, if what is socially safe vis-à-vis adults is not socially safe vis-à-vis other children (e.g. $r_{c c}>1 / 2$ and $r_{c p}<1 / 2$ ), an altruistic parent will take into account the child's concern toward his peers and will thus have a weaker incentive to distort his behavior. The other difference with the result in the previous section is the presence of the parameter $\delta$. As intuition suggests, the distortion increases in the child's exposure to parental behavior.

Consider now the sensitivity of the child's payoff to the state of nature, $l_{\theta}$ and assume first that the social pressure on the parent $\left(\bar{\omega}_{p}\right)$ is negligible. Then, an increase in $l_{\theta}$ reduces the absolute value of the distortion. Intuitively, a higher $l_{\theta}$ increases the importance for the child of "getting $\theta$ right". Signaling about the state of nature becomes more important relative to norm-signaling. As a result, as $l_{\theta}$ becomes larger, the parent wants to move his cutoff toward the optimal cutoff when all that matters is the state of nature (i.e. $k=0$ ). This is evident by looking at the cutoff expression (13). An increase in $l_{\theta}$ reduces both the weight of the norm-signaling term and the weight of the term $-\beta$.

Another effect is however present when the social pressure on the parent is non-negligible. The weight of the term $\bar{\omega}_{p}\left(\bar{r}_{p}-1 / 2\right)$ in (13) is inversely related to $l_{\theta}$. Intuitively, as the child becomes more exposed to the state of nature, it becomes also less crucial for the parent to conform to others. As a result, the importance of the parent's own socially safe choice is decreased. When the parent's overall safe choice is misaligned with that of the child (e.g. $\bar{r}_{c}<1 / 2$ and $\bar{r}_{p}>1 / 2$ ), this indirect effect may outweigh the direct effect, so that the normsignaling bias is reinforced. As a result, higher sensitivity to the state of nature of the child's payoff has ambiguous effects on the norm signaling bias.

Finally, to fully appreciate the welfare consequences of the norm-signaling bias, consider the case of positive externalities $(e>0)$. In the absence of signaling concerns, the propensity of adults to choose the high action - i.e. the action providing the positive externality - will be generally too low. In other words, $\theta^{n s}$ will be higher than the socially optimal threshold. In these cases, if the top action is socially safe for children, the norm signaling bias will move the equilibrium cutoff closer to the social optimum. We will see in Section 4.3 an application where parents choose to provide a public good (pro-social values) to signal to their children that a 
norm for honesty is widespread. ${ }^{21}$ That said, it is easy to come up with examples where the reverse occurs, so that the norm-signaling bias exacerbates negative externalities.

One might perhaps wonder whether the norm signaling bias is an artefact of equilibrium selection. The next result provides sufficient conditions for uniqueness.

Proposition 3. When $l_{\theta}>0, \delta l_{\theta}<1$, and $D$ is sufficiently large, the equilibrium characterized in Proposition 2 is the unique equilibrium of the game.

Proof. See supplementary material.

The conditions $\delta l_{\theta}<1$ and $D$ large enough ensure that there exist signal realizations such that either action is dominant for adults. This rules out perverse equilibria where children always do the opposite of what parents do, so that, when $\theta_{t}$ is large, adults end up choosing the low action to induce their child to choose the top action. Adding $l_{\theta}>0$ ensures that, when peer pressure among children is particularly strong, there is no equilibrium where children only focus on coordinating among themselves, ignoring adult behavior.

\section{Applications}

We now discuss a few examples that illustrate the socio-economic implications of the normsignaling bias. To better isolate the effect of norm-signaling, we will cast each application in terms of a two stage signaling game with no conflict of interest. That said, it is not difficult to see how in each case the interests of parents and their children may be imperfectly aligned.

\subsection{Attitudes toward sex and hypocrisy}

Parental attitudes toward sex are obviously important determinants of the sexual behavior of children. A direct and obvious channel through which parental attitudes affect child behavior is the fear of confronting disapproval by one's parent. In this section, we discuss an alternative channel where the parent acts as a vehicle for societal pressures to conform.

Consider a two stage game where parents first have to decide whether to take a liberal attitude toward sex $(a=1)$ or a conservative attitude $(a=0)$. Taking a conservative attitude

\footnotetext{
${ }^{21}$ The intuition for this result is similar to that given in Bernheim and Thomadsen (2005) for the case of selfsignaling. Different from their model, the current setup allows to predict the circumstances that allow cooperation to emerge. This only happens when, by choosing the socially optimal action, an adult encourages his child to select the socially safe choice.
} 
involves disapproving of "deviant" behavior. For concreteness, suppose that this takes the form of stigmatizing youngsters who have sex before marriage. ${ }^{22}$ In the second stage, children choose whether to engage in pre-marital sex $(\alpha=1)$ or to abstain $(\alpha=0)$. The payoff from abstinence is set equal to zero. Engaging in pre-marital sex yields a direct utility,

$$
\tilde{v}(x ; \theta)=\theta-\omega_{c p}(1-x),
$$

where $1-x$ is the share of adults who take a conservative attitude. The first term, $\theta$, represents the direct net benefits (or costs) from pre-marital sex. These clearly reflect the direct utility from the act or from a better informed choice of partner, the quality and availability of contraceptives, the risk of unwanted pregnancies and of sexually transmitted diseases. The second term, $\omega_{c p}(1-$ $x$ ), represents the costs of shaming/social boycott coming from society. These increase as more adults take a conservative attitude toward sex.

Parents' direct payoff from taking a conservative attitude is set equal to zero. Their direct expected payoff from liberalism reflects their assessment of the intrinsic benefits/costs of premarital sex, $\theta_{i}$. We say that an adult has conservative beliefs if, on balance, he thinks that pre-marital sex is a bad thing $\left(\theta_{i}<0\right)$. We say that an adult has liberal beliefs in the opposite case $\left(\theta_{i}>0\right)$. We set for simplicity $\beta=0$, so that there is no conflict of interest between parent and child. Our results would simply become more extreme if parents had a bias against pre-marital sex (for instance because they do not fully internalize the utility the child derives from the act). ${ }^{23}$ We also assume that children form their beliefs by looking at parental behavior with probability $\delta \leq 1$. Total parental utility is provided in Table 2 .

If children were able to directly observe $x$, their choice between sex and abstinence would be independent of parental attitudes. It is then clear that parents would choose conservatism whenever they have conservative beliefs $\left(\theta_{i}<0\right)$ and would choose liberalism otherwise. As we now argue, things change when children are unable to observe $x$, so that norm-signaling occurs.

\footnotetext{
${ }^{22}$ Note that we are implicitly assuming that parents can commit ex ante to shame future deviant behavior. This may for instance be accomplished by choosing an appropriate identity (liberal, conservative), in the vein of Akerlof and Kranton (2000). Alternatively (at the cost of additional complication) we could have constructed a model where different parent-child pairs move sequentially, and where each parent may send a signal to his child by shaming/not shaming deviant children from the previous cohort.

${ }^{23}$ We consider a setup where parents who fail to shame deviants do not incur any direct sanction themselves. As a result, we abstract from social pressure on parents, so that $\boldsymbol{\omega}_{p}=0$. This is meant to clarify that signaling motives alone may be sufficient to induce excessive stigmatization.
} 


\begin{tabular}{lccc}
\hline & & \multicolumn{2}{c}{ Child's choice } \\
& & Abstinence & Pre-marital sex \\
\hline \multirow{2}{*}{ Parental attitude } & Conservative & 0 & $\delta\left[\theta-\omega_{c p}(1-x)\right]$ \\
& Liberal & $\theta$ & $\theta+\delta\left[\theta-\omega_{c p}(1-x)\right]$ \\
\hline
\end{tabular}

Table 2: Total parental utility (terms in brackets represent parent's utility from the child's choice).

It is immediate to check that this setup is a special case of the one seen in Section 3 having set $r_{c p}=l_{\theta}=1$ and all other parameters (except $\omega_{c p}$ and $\delta$ ) equal to zero. We thus have,

Result 1. Adults adopt a liberal attitude if $\theta_{i} \geq \theta^{*}=\left(\omega_{c p} / 2\right)(\delta / 1+\delta)$ and adopt a conservative attitude otherwise. Children engage in pre-marital sex when observing an adult with a liberal attitude and choose abstinence (i.e. the socially safe choice) otherwise.

Figure 1 illustrates adults' behavior.

Parents with conservative

beliefs
Parents with liberal

beliefs

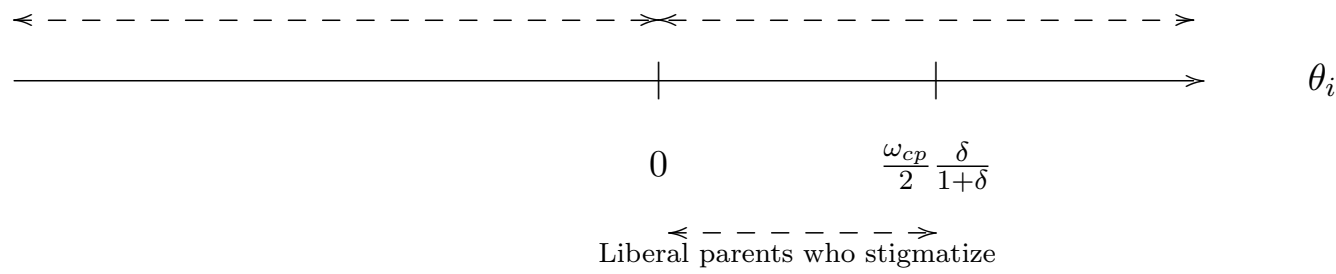

Figure 1: Attitudes toward sex

Since abstaining from pre-marital sex does not attract any disapproval, abstinence is socially safe for children. The signaling distortion thus takes the form of parents adopting a conservative attitude more often than they would otherwise. Some parents will take a conservative attitude even if they have liberal beliefs, i.e. even when they think that, on balance, pre-marital sex is a good thing. This is done to induce their children to adopt a socially safe behavior.

Interestingly, for $\epsilon$ sufficiently small, there exist realizations of $\theta$ such that the attitudes of all parents are prompted by hypocrisy: they all have liberal beliefs, but still take a conservative attitude. Even more, it may happen that all parents know that all other parents have liberal beliefs, but they still choose to be conservative. Intuitively, the need to signal to children that there is a norm against pre-marital sex forces parents to follow the norm, thus helping to preserve it. To see how this can happen, suppose that $\theta \in\left(3 \epsilon, \theta^{*}-\epsilon\right)$. A parent with signal $\theta_{i}$ knows that any other parent necessarily has $\theta_{j} \geq \theta_{i}-2 \epsilon$. Hence, the least liberal parent - with signal 
equal to $\theta-\epsilon-$ knows for sure that all other parents must have $\theta_{j} \geq \theta-3 \epsilon>0$. All other parents clearly know that the lowest $\theta_{j}$ is above that level. ${ }^{24}$ Yet, all parents choose to take a conservative attitude. It is also interesting to note that hypocrisy is reduced as children become more likely to obtain information from sources beyond parental control (lower $\delta$ ).

There is evidence to suggest a degree of hypocrisy in parents. Using questionnaire data, Newcomer and Udry (1985) show that teenagers substantially underestimate their mothers' liberalism over sex issues. This is consistent with the idea that parents may strategically adopt a more conservative attitude than their personal inclinations, in order to influence their children's behavior.

\subsection{Work ethic and collective reputation}

Models of statistical (i.e. information driven) discrimination (see e.g. Coate and Loury, 1993) typically rely on a collective reputation mechanism to generate self-fulfilling prophecies. If members of a minority expect to be discriminated because of negative stereotypes, they will have less incentive to invest in skills, thus confirming those stereotypes. A possible shortcoming of these theories is that they do not provide an account of beliefs formation. Similar to Bénabou and Tirole (2006), we propose a theory where parents, by choosing whether or not to endow their children with strong work norms, strategically manipulate their "belief in a just world".

We consider a simplified two stage adaptation of Coate and Loury (1993), which is augmented with an explicit account of belief formation. Parents first choose whether to instil strong or weak work norms into their children. We model work norms as a commitment to exert effort. Once employed, children with strong work norms exert high effort, while children with weak work norms exert low effort. There are two types of jobs: skilled and unskilled. The unskilled job pays $w \geq 0$ if the worker exerts low effort and $w+\theta$ if he exerts high effort. The skilled job pays the same as the unskilled job plus a skill premium $\Delta w>0$ (See Table 3). The state of nature $\theta$ captures the extent to which effort on the job is rewarded net of its cost. We will interpret it as the incentive system of society.

Workers can access the skilled job only by investing in skills in their youth. The investment costs $c>0$, with $c<\Delta w$, and is imperfectly observed by employers. We assume that if the

\footnotetext{
${ }^{24}$ While it is common knowledge that all have liberal beliefs, it is not necessarily common knowledge that all know that all have liberal beliefs. This would require the stronger condition $\theta>5 \epsilon$. In turn, common knowledge of the fact that all know that all know that all have liberal beliefs requires $\theta>7 \epsilon$ and so on.
} 


\begin{tabular}{cccc}
\hline & & \multicolumn{2}{l}{ Child } \\
& & Not invest & Invest \\
\hline \multirow{2}{*}{ Parent } & Weak norms & $w$ & $w+[x \Delta w-c]$ \\
& Strong norms & $w+\theta$ & $w+\theta+[x \Delta w-c]$ \\
\hline
\end{tabular}

Table 3: Total parental utility (terms in brackets reflect parent's utility from child's choice).

worker has not invested, the employer will receive a low signal for sure and allocate the worker to the unskilled job. If the worker has invested, the employer will observe a high signal (and allocate the worker to the skilled job) with probability $x$, where $x$ is the aggregate share of workers with strong work norms in the group. With the complementary probability, $1-x$, the employer receives a low signal and allocates the worker to the unskilled job. ${ }^{25}$

An interesting feature of this setup is that oblique complementarities arise indirectly, from the collective reputation mechanism. An employer is more likely to notice that a worker is skilled if the worker belongs to a group with high work ethic. Hence, employers partly form their beliefs based on aggregate information - namely stereotypes about the pervasiveness (or lack thereof) of work ethic in the group. This implies that, if the group is perceived as lacking work ethic, each worker will face worse job prospects (even if he has invested), and vice-versa. Just as in Coate and Loury (1993), these stereotypes will prove to be relevant since in equilibrium a group's work ethic actually reflects its aggregate level of human capital.

Since parents' choices of work norms are correlated - they all depend on the net return from effort $\theta$ - a child's own work norm is a good indicator of the work norms of other group members and, accordingly, of whether it is worth to invest in skills. A child with a strong work norm not only exerts higher effort once he gets a job, he is also more inclined to invest in order to land a better job. In turn, this implies that stereotypes on a group's work ethic are informative about the group's human capital investment.

This setup is a special case of the full model if we set $r_{c p}=c / \Delta w, \omega_{c p}=\Delta w, \delta=1$, and all other parameters equal to zero. ${ }^{26}$ It follows that,

\footnotetext{
${ }^{25}$ We do not model employers' objectives explicitly. The behavior we assume is however compatible with employers' equilibrium behavior in a model à la Coate and Loury (1993) where labor demand is perfectly elastic but employers face costs whenever job and worker characteristics (skilled/unskilled) are mismatched.

${ }^{26}$ It is worth noting that, while $\theta$ determines the welfare of children with a strong work norm, their net payoff from investing does not depend on the state of nature in this simple setting. This explains why $l_{\theta}=0$. Qualitatively similar results would obtain if the incentives to invest were linearly increasing in $\theta-$ so that $l_{\theta}>0$. Moreover, the assumption that children with strong norms are pre-committed to exert effort is only needed for consistency with the model seen in Section 3, but is not necessary for the results.
} 
Result 2. Parents instil a strong work norm in their child if $\theta_{i} \geq \theta^{*} \equiv c-\Delta w / 2$, and a weak work norm otherwise. Children with strong work norms invest in skills, while children with weak norms do not.

This result has implications both for socio-economic theories of work ethic and for theories of information-based discrimination. The nature of the signaling distortion is determined by the ratio $c / \Delta w$. We say that a group lives in a low mobility environment if access to education is costly relative to the skill premium $\left(c>\Delta w / 2\right.$, or, equivalently, $\left.r_{c p}=c / \Delta w>1 / 2\right)$. We say that it lives in a high mobility environment if the reverse occurs. Since $r_{c p}>1 / 2$, in a low mobility environment, not investing in skills is the socially safe choice. A parent may thus choose a weak work norm even if the net return from effort is positive. Intuitively, the parent expects that, since other children of the group will lack work ethic, his child will be at a disadvantage. A weak work norm is optimal because it signals to the child that he should not waste resources in trying to climb the social ladder. This implies that weak work norms may persist even when incentives (e.g. the benefit system) are designed to make effort profitable. By contrast, in a highly mobile environment, investing is socially safe. Strong work norms may thus persist even in the presence of poorly designed incentives that favor low effort.

Notice that a child with a strong work norm has a positive outlook on society and its fairness. He expects that investing in skill will ultimately be rewarded. By converse a child with a weak work norm expects discrimination to be rife. Hence, at the micro level, the model highlights that the work norms one inherits from his family background are important determinants of beliefs. At the aggregate level, the beliefs of a group (and thus its chances of social advancement) will ultimately be determined by the interaction between its perception of the incentive system $(\theta)$, and other characteristics of the environment like the accessibility of the education system vis-á-vis the skill premium.

\subsection{Trust, values, and crime}

Why do parents choose to pass pro-social values to their children? Why does this happen even in societies with weak or inefficient legal systems, which fail to reward pro-social behavior or even encourage opportunism? These are important questions given that pro-social norms are often all that prevents societies with weak or corrupt governance from unravelling. ${ }^{27}$

\footnotetext{
${ }^{27}$ Recent economic literature on crime (see e.g. Silverman, 2004) also raises the opposite puzzle. Why do we observe anti-social behavior even in environments where this is manifestly sub-optimal?
} 
The following application builds on the idea - introduced in Adriani and Sonderegger (2009) - that the types of values instilled by a parent in his child provide information about the dominant norm in society. A child raised with pro-social values will infer that pro-social values are widespread and will thus be more optimistic about society (and therefore more inclined to trust others) than a child who has inherited anti-social values.

Consider the following setting. Each child can behave either in a trusting or distrustful manner. He can also be either trustworthy or untrustworthy ("cheat"). Trusting yields a material payoff $\tau x-q(1-x)$, where $x$ is the unobserved share of trustworthy children and $(1-x)$ the share of cheaters. Hence, $\tau>0$ is the marginal benefit of an additional trustworthy individual and $q>0$ is the marginal cost of an additional cheater. The payoff from being distrustful is normalized to zero.

The direct payoff from being trustworthy is also set equal to zero. The payoff from cheating is $b \xi-\theta$, where $\xi \in[0,1]$ is the unobserved share of trusting children. We assume that $b>0$, so that the rewards from cheating increase with the share of trusting individuals. Intuitively, a larger share of trusting individuals means a larger pool of potential victims. The state of nature $\theta$ can be interpreted as the extent to which the institutional environment may or may not punish cheaters. This may for instance reflect the quality of governance, the legal system, or the effectiveness of enforcement.

Having observed their private signal $\theta_{i}$ about $\theta$, parents choose whether to give pro-social or anti-social values to their child. A child with pro-social (anti-social) values is committed to trustworthiness (cheating). ${ }^{28}$ The child can however choose whether to trust or not. Table 4 illustrates the payoffs.

\begin{tabular}{lccc}
\hline & & Child & \\
& & Trusting & Distrustful \\
\hline \multirow{2}{*}{ Parent } & Pro-social values & {$[\tau x-(1-x) q]$} & 0 \\
& Anti-social values & $b \xi-\theta+[\tau x-(1-x) q]$ & $b \xi-\theta$ \\
\hline
\end{tabular}

Table 4: Total parental utility (terms in brackets reflect parent's utility from child's choice).

The question we ask is the following. Under what conditions, if any, would a parent want to raise his child as pro-social? In the absence of signaling motives, parents may want to give

\footnotetext{
${ }^{28}$ Notice however that this would be consistent with the child's equilibrium behavior so long as $\theta$ is unobservable. We assume commitment to keep exposition simple and to make the setup comparable with what we have seen in the previous sections.
} 
pro-social values only when the consequences of cheating $(\theta)$ are negative enough to counteract the material advantages $(b \xi)$. It is then clear that no parent with $\theta_{i}<0$, i.e. believing that cheating pays, would ever pass pro-social values to his child. ${ }^{29}$

Consider now what happens in this setup if we introduce norm signaling. We argue that, if the potential rewards from trust are sufficiently large, so that $\tau>b+q$, a parent may raise his child as pro-social even if he believes that the institutional environment rewards untrustworthiness $(\theta<0)$. Symmetrically, if $\tau<b+q$, a parent may raise his child as anti-social even when cheating is suboptimal. The first step is to recognize that the share of trustworthy children coincides with the share of trusting children. In particular, whenever $\epsilon$ is sufficiently small relative to $D, \xi=x$ in any threshold equilibrium. ${ }^{30}$

Notice now that, since all individual players are small, there is no loss of generality in imposing $\xi=x$ at the outset. It is convenient to work directly with these "reduced form" payoffs. The inclusive payoff from pro-social values is thus

$$
\underbrace{-(b x-\theta)}_{\text {Forgone dishonesty rents }}+\underbrace{[(\tau+q) x-q] \times \Delta \alpha}_{\text {Utility from trust }} .
$$

Setting $\omega_{p p}=-b, \omega_{c p}=\tau+q, r_{c p}=q /(\tau+q), \delta=1$ and all other parameters equal to zero shows that the "reduced form" setup is isomorphic to that of Section 3. A peculiar feature of this setting deserves special attention. Since $b>0$, we have $\omega_{p p}<0$. In words, parental actions (to raise their child as pro-social/anti-social) are strategic substitutes. Intuitively, since (in equilibrium) pro-social children are trusting, a parent's choice to raise his child as pro-social increases the stock of trusting children. In turn, this increases the expected rents obtained from cheating, thus weakening the incentives, for other parents, to raise their children as pro-social. In order to ensure that Assumption A2 (supermodularity of inclusive payoff) is satisfied, we

\footnotetext{
${ }^{29}$ Formally, if children could observe the share of pro-social agents $x$, a child's choice to be trusting or not would be independent of his values. A child will be trusting if the proportion of pro-social individuals is sufficiently large $(x \geq q /(q+\tau))$ and will not trust otherwise. Consider now parents. If they choose to endow their child with pro-social values their child cannot benefit from cheating. This costs $\xi b-\theta$. Hence, the expected net payoff from passing pro-social values of a parent with private signal $\theta_{i}$ is $\theta_{i}-b E\left(\xi \mid \theta_{i}\right)$.

${ }^{30} \mathrm{~A}$ child will trust if and only if he expects the share of trustworthy children $x$ to be at least $q /(\tau+q)$. As shown in the proof of Lemma 1 (see supplementary material), when parents use a sufficiently interior cutoff $k$,
}

$$
E(x \mid \text { dishonest }) \leq \frac{\epsilon}{k+D}, E(x \mid \text { honest }) \geq 1-\frac{\epsilon}{D-k}
$$

Hence, for $\epsilon$ small, the share of trusting children coincides with the share of trustworthy children. 
need to impose $\tau+q>b$.

Result 3. Assume $\tau+q>b$. Then, parents endow their children with pro-social values if $\theta_{i} \geq \theta^{*}=(q+b-\tau) / 2$ and with anti-social values otherwise. Children choose to be trusting if and only if they are pro-social.

Notice that, for $\tau>q, r_{c p}<1 / 2$. In other words, when the positive externality created by an additional trustworthy child outweighs the negative one produced by an additional cheater, trusting is the socially safe choice. If the potential rewards from trusting are large enough $(\tau>q+b)$, the norm signaling bias generates a negative cutoff $\left(\theta^{*}<0\right)$. As a result, there exist $\theta_{i}<0$ such that parents want to pass pro-social values to their children even if cheating appears to be materially optimal. They do this to signal that they are optimistic about the share of honest individuals in society, so that their child will trust. Since other parents do the same, the pro-social norm becomes self-fulfilling and the actual rewards from trusting outweigh the forgone rents from cheating. For $\tau<q, r_{c p}>1 / 2$, so that the socially safe choice is not to trust. As a result, the cutoff is always strictly positive. In this case, a "street culture" may emerge, whereby parents instil anti-social values even when cheating does not pay. Again, the reason they do this is to signal that trusting others is a bad idea. Since other parents reason in a similar way, this prophecy becomes self-fulfilling.

Adriani and Sonderegger (2009) study this problem in a setup with homogeneous beliefs and find that there is a continuum of equilibria where parents choose pro-social values for their children. The current setup delivers clear comparative statics on the cutoff $\left(\theta^{*}=(q+b-\tau) / 2\right)$. Parents' propensity to raise their children as pro-social depends not only on the marginal benefit from dishonesty $b$, which is somehow expected, but also on the marginal benefits and costs from trusting $(\tau$ and $q)$. This makes the distribution of pro-social preferences in the population depend on the (observable) returns from participating in economic exchanges.

\section{$5 \quad$ Further extensions and robustness}

\subsection{Persistence of social norms}

Many observers perceive culture as "slow moving". ${ }^{31}$ While there is abundant anecdotal evidence of adults adopting political or social attitudes radically different from those predominant in their

\footnotetext{
${ }^{31}$ The Marshall lecture at the 2007 meeting of the European Economic Association focused on the issue of cultural persistence (see Guiso et al., 2008).
} 
youth, many people feel uneasy to depart from the social norms they have internalized at an early age. The question is then, can a model in which cultural transmission is modelled as information transmission accommodate the slow moving culture view? In order to extend the model in this direction, a natural starting point is to assume that adults are partially "backward looking". Similar to Acemoglu and Jackson (2015), we allow generation $t$ adults to have a preference for conforming to the predominant norm among $t-1$ adults $\left(x_{t-1}\right)$. In the supplementary material, we characterize the threshold equilibrium of this game and provide conditions for existence. Assuming for simplicity that $x_{t-1}$ is publicly observable at time $t$, the (time varying) adults' cutoff in any period $t, k\left(x_{t-1}\right)$, is decreasing in its argument. This implies that social norms tend to persist across generations. For instance, a temporary negative shock like the emergence of a new sexually transmitted disease (a low realization of $\theta$ ) may establish in the short run a norm for conservatism (a low $x_{t}$ ). The norm might in principle persist long after the direct short run effects of the shock have withered away (e.g. because the population has developed immune resistance to the disease). Changing the norm may eventually require a large positive shock in the other direction (e.g. a major improvement in contraception technology). That said, we show that the static properties of the model are unchanged so that, ceteris paribus, adults will still tend to be biased toward behavior that is socially safe.

\subsection{Costless communication in parent-child relationships}

A possible objection to our setup is that it does not explicitly allow parents to use other methods except their own actions to transmit information to children. What might happen if we allow for direct (and possibly costless) communication between parents and children?

In the baseline model, we saw that the total signaling distortion could be split into two components

$$
\text { total distortion }=-\beta+\text { norm-signaling bias. }
$$

It is clear that allowing for cheap talk would generally not eliminate the conflict of interests component $(\beta)$ of the distortion. However, since the norm signaling component does not arise from a conflict of interests, it is fair to ask whether it might disappear if we allowed for costless communication. We offer two remarks on this. First, at an informal level, once a society has settled on an equilibrium without communication, it might be quite difficult for parental cheap talk to destabilize it. Consider for instance a parental deviation consisting in choosing the low 
action and giving instructions to the child to choose the top action. For this to work, the child needs to hold conflicting beliefs about his parent. First, he has to believe that his parent is fairly representative of the adult population (otherwise there is no point in paying attention to what he does or says in the first place). However, he also needs to believe that his parent is somewhat special (i.e. the deviation is truly unilateral). If the child suspects that other parents might behave like his own, he might have no incentive to switch to the top action.

The above argument is essentially an equilibrium selection argument. There is however a deeper point that applies to any equilibrium of the (cheap talk augmented) game. In the previous sections we focused for convenience on the case where $\beta=0$. In this case, there exist equilibria with parent-child communication where parents do not distort their behavior. However, $\beta$ is unlikely to be exactly zero in most empirically relevant situations. We show in the Appendix that, whenever $\beta \neq 0$, the norm-signaling term in (19) is also different from zero in all equilibria of the game - i.e. even allowing for meaningful communication. In other words, equilibria where the total distortion is equal to $-\beta$ exist only if $\beta$ is zero. ${ }^{32}$ Depending on the details, the norm-signaling bias may exacerbate, mitigate, or even totally offset the distortion caused by the conflict of interests. This however implies that norm-signaling concerns are generally non-neutral - since they affect equilibrium behavior above and beyond the distortion directly caused by the conflict of interests. The intuition for this result is straightforward. If a parent could use cheap talk to avoid the distortion due to norm signaling, he would use it also to avoid the distortion due to the conflict of interests. In other words, if the parent could "talk" his child into choosing a particular action, he would exploit communication to deceive the child when their interests are misaligned. However, this is clearly incompatible with equilibrium behavior. We formalize this non-neutrality result in the supplementary material.

\section{Concluding Remarks}

Many models of culture emphasize how societies may endogenously value some personal characteristics or activities beyond their intrinsic economic value (see e.g. Fang, 2001, Mailath and Postlewaite, 2006). One question that these models are not designed to tackle is why some activities end up being overvalued while others do not. For instance, is there anything inherently special in an Oxford accent? Addressing this problem in a theoretically compelling way (i.e.

\footnotetext{
${ }^{32}$ Even in this case, though, the equilibrium we focused on in the previous sections would continue to exist.
} 
without assuming the result) is of course extremely difficult. While our paper does not provide a direct answer to the question, it nevertheless contributes an intermediate step that may prove useful in answering it. The very nature of the process of cultural transmission implies that whether an activity is socially safe or not may shape attitudes toward that particular activity. While in some cases different activities may be perfectly symmetric (e.g. driving on the left or on the right), our applications suggest that, generally, an asymmetry will be present. In these cases, the criterion of social safety allows to identify a precise activity. Our theory suggests that society will be more likely to display a favorable attitude toward that activity. Understanding the conditions that make an activity socially safe/unsafe could thus provide a valuable clue to understanding which activities might be valued beyond their intrinsic economic worth.

\section{References}

Acemoglu, D. And M.O. Jackson (2015) "History, Expectations and Leadership in the Evolution of Social Norms." Review of Economic Studies, 82, 423-456.

Adriani F. And S. Sonderegger (2009) "Why do Parents Socialize Their Children to Behave Pro-socially? An Information-based Theory." Journal of Public Economics 93, 1119-1124.

Adriani F. And S. Sonderegger (2015) "Trust, Trustworthiness and the Consensus Effect: An Evolutionary Approach." European Economic Review 77, 102-116.

Akerlof, G. And R. Kranton (2000) "Economics and Identity." Quarterly Journal of Economics $115,715-753$.

Arrow, K. J. (1973) "The Theory of Discrimination," in O. Ashenfelter and A. Rees (eds.) Discrimination in Labor Markets, Princeton University Press.

Bénabou, R., and J. Tirole (2006) "Incentives and Prosocial Behavior." American Economic Review 96, 1652-1678.

Bénabou, R., And J. Tirole (2011) "Laws and Norms." NBER Working Paper 17579.

Ben-ner, A., List J.R. , Putterman, L. And A. Samek (2015) "Learned Generosity? A Field Experiment with Parents and their Children." CESR-Schaeffer Working Paper.

Bernheim, B.D., (1994) "A Theory of Conformity." Journal of Political Economy 102, 841-877. Bernheim, B.D. And R. Thomadsen (2005) "Memory and Anticipation." Economic Journal, $115,271-304$. 
Bisin, A., AND VERDier, T. (2001). "The economics of cultural transmission and the dynamics of preferences." Journal of Economic Theory, 97, 298-319.

Bisin, A., AND Verdier, T. (2010). The economics of cultural transmission abd socialization. Discussion paper, NBER Working Paper 16512.

Boyd, C.F. And P.J. Richerson (1985), Culture and the Evolutionary Process, University of Chicago Press, Chicago.

Carlsson, H. and E. van Damme (1993) "Global Games and Equilibrium Selection." Econometrica 61, 989-1018.

Cavalli-Sforza L.L. And M.W. Feldman (1981). Cultural transmission and evolution: a quantitative approach. Princeton University Press.

Coate, S. And G. C. Loury (1993) "Will Affirmative-Action Policies Eliminate Negative Stereotypes?" American Economic Review 83, 1220-40.

Ellingsen, T., and M. Johannesson (2008) "Pride and Prejudice: The Human Side of Incentive Theory." American Economic Review 98, 990-1008.

FANG, H., (2001) "Social Culture and Economic Performance." American Economic Review 91, 924-937.

Guiso, L., Sapienza, P., And L. Zingales (2008). "Alfred Marshall lecture. Social capital as good culture." Journal of the European Economic Association 6, 295-320.

Hauck, E. And M. Saez-Martí (2002) "On the cultural transmission of corruption." Journal of Economic Theory 107, 311-335.

Hermalin, B.E. (1998) "Toward an Economic Theory of Leadership: Leading by Example." American Economic Review 88, 1188-1206.

Houser, D., List, J.R., Piovesan, M., Samek, A. And J. Winter (2016) "Dishonesty: from parents to children." European Economic Review 82, 242-254.

Kandori, M., Mailath, G.J., And R. Rob (1993) "Learning, Mutation, and Long Run Equilibria in Games." Econometrica 61, 29-56.

Kuran, T. And W.H. Sandholm (2008) "Cultural integration and its discontents." Review of Economic Studies 75, 201-228.

LeVy, G. AND R. RAZIN (2012) "Religious beliefs, religious participation and cooperation." American Economic Journal: Microeconomics, 4, 121-151. 
LEvy, G. AND R. RAZIN (2014) "Rituals or good works: social signalling in religious organizations" Journal of European Economic Association, forthcoming.

Mailath, G.J., And A. Postlewaite (2006) "Social Assets." International Economic Review 47, 1057-1091.

Marks, G. AND N. Miller (1987) "Ten years of research on the false-consensus effect: An empirical and theoretical review." Psychological Bulletin 102, 72-90.

Morris, S. AND H. S. Shin (1998) "Unique Equilibrium in a Model of Self-fulfilling Currency Attacks." American Economic Review 88, 587-597.

Morris, S., AND H.S. Shin (2003) Global Games: Theory and Applications, in M. Dewatripont, L. Hansen and S. Turnovsky (eds.) Advances in Economics and Econometrics, the Eighth World Congress, Cambridge University Press.

NewComer, S.F. ANd J.R. Udry (1985) "Parent-Child Communication and Adolescent Sexual Behavior." Family Planning Perspectives 17, 169-174.

Ross, L., Green, D and P. House (1977) "The false consensus effect: An egocentric bias in social perception and attribution processes" Journal of Experimental Social Psychology 13, 279-301.

Rubinstein, A. And Y. SAlant (2016) "Isn't everyone like me?: On the presence of self-similarity in strategic interactions." Judgment and Decision Making, 11, 168-173.

Saez-Martí, M. And A. SjöGren (2008) "Peers and Culture." Scandinavian Journal of Economics $110,73-92$.

Saez-Martí, M. and Y. Zenou (2012) "Cultural Transmission and Discrimination." Journal of Urban Economics, 72, 137-146.

Silverman, D., (2004) "Street Crime and Street Culture." International Economic Review 45, $761-786$.

SliwkA, D. (2006) "Trust as a Signal of a Social Norm and the Hidden Costs of Incentive Schemes." American Economic Review 97, 999-1012.

Tabellini, G. (2008) "The Scope of Cooperation: Values and Incentives." Quarterly Journal of Economics 123, 905-950. 